\title{
Évaluation de l'impact radiologique de différentes voies de gestion des déchets TFA
}

\author{
B. CESSAC*, J.M. PÉRÈS*
}

(Manuscrit reçu le 14 janvier 2000, révisé le 9 juillet 2000, accepté le 23 août 2000)

RÉSUMÉ Deux orientations se dessinent aujourd'hui pour la gestion des déchets TFA, le stockage en site de surface d'une part, et la mise en place de filières d'élimination ou de valorisation d'autre part. Les ordres de grandeur des conséquences radiologiques que pourraient induire ces deux options sont exposés au travers de deux exemples, à titre d'illustration plutôt que de comparaison. Pour le stockage, l'impact radiologique est présenté au travers d'un cas générique, assorti des incertitudes découlant de la variabilité des paramètres liés au concept, à la géosphère et à la biosphère. Dans le cas des filières, la méthodologie d'évaluation appliquée s'appuie sur la description poste par poste des processus industriels d'élimination ou de valorisation, ainsi que des conséquences pour la population. Sont ainsi présentées l'élimination ou la valorisation d'huiles usagées TFA et l'élimination après vitrification de déchets amiantifères ou de calorifuges TFA.

ABSTRACT Radiological impact assessment of very low-level radioactive waste management pathways.

Two approaches are currently being adopted for the management of very low-level waste (VLLW): disposal in surface facilities and, for some categories of VLL waste, the implementation of treatment and utilisation channels. In this paper, the orders of magnitude of the radiological consequences which may result from these two options are discussed, in a matter of a description rather than a comparison. As regards disposal, radiological impact is presented through a generic case, along with the uncertainties stemming from the variability of the parameters associated with its design, as well as those associated with the geosphere and the biosphere. In the case of treatment and utilisation channels, such as disposal or recycling of VLL waste oils or VLL wastes containing asbestos, the assessment of radiological consequences deals with the public exposure and with the occupational exposure on the basis of a stageby-stage breakdown of the industrial process.

\section{Introduction}

Avec des activités s'échelonnant de 1 à $100 \mathrm{~Bq} \mathrm{~g}^{-1}$ environ, suivant les radionucléides, les déchets de très faible activité, ou TFA forment une catégorie intermédiaire entre les déchets radioactifs de catégorie A et les déchets conventionnels. Les principales quantités de déchets TFA sont générées dans les installations nucléaires de production, tout au long du cycle du combustible. Pour

\footnotetext{
* Institut de protection et de sûreté nucléaire, DPRE/SERGD/LESTS, BP 6, 92265 Fontenay-aux-Roses Cedex, France.
} 
les seules installations d'Électricité de France, la maintenance et l'exploitation des réacteurs et surtout les futurs chantiers de démantèlement génèreront plus de 700000 tonnes de ces déchets (Hilmoine, 1997).

Les TFA présentent une activité massique faible ne justifiant pas, pour leur stockage, de mesures de protection telles que celles développées pour le centre de stockage de l'Aube (CA), destiné aux déchets de faible et moyenne activité. Néanmoins, le caractère radioactif des déchets TFA n'autorise pas leur banalisation en centre d'enfouissement technique pour déchets conventionnels ou dans toute autre filière industrielle. Un mode de gestion spécifique est donc nécessaire.

La démarche actuelle proposée par les autorités de sûreté nucléaire, a pour objet d'aider le producteur à réduire les volumes de déchets, si possible, à assurer leur valorisation ou à permettre leur élimination et in fine, leur stockage dans des conditions garantissant la protection du public et de l'environnement. En application de cette démarche et à la demande des pouvoirs publics ou des exploitants, l'IPSN a réalisé plusieurs études d'où sont tirés les exemples synthétisés dans le présent article :

- une estimation de la variabilité associée à l'évaluation de l'impact radiologique d'un stockage en fonction des incertitudes conceptuelles, géologiques et environnementales ( $c f$. paragraphe 3 );

- une évaluation, sur le plan radiologique, de différentes filières de recyclage ou d'élimination de déchets TFA spécifiques, huiles usagées ou déchets amiantifères ( $c f$. paragraphe 4 ).

\section{Mode de gestion adoptée pour les déchets TFA}

Les autorités de sûreté françaises proposent aujourd'hui une politique de gestion des déchets TFA basée sur la responsabilité totale des producteurs et la traçabilité des déchets depuis leur origine jusqu'à leur destination finale (DSIN, 1997). La mise en place de cette politique repose sur la réalisation, par les exploitants du cycle du combustible, d' « études-déchets ». Ces études imposent en premier lieu de réaliser un zonage des installations, lorsque celles-ci n'en bénéficient pas, en distinguant des zones conventionnelles au sein desquelles tout déchet pourrait être considéré comme non contaminé par opposition à d'autres zones qualifiées de nucléaires. Ce zonage, complété par un tri à la source, doit permettre la distinction de catégories de déchets de niveaux de radioactivité différents, en vue d'une gestion ultérieure spécifique. La comparaison de différentes solutions potentielles de gestion devrait permettre de sélectionner et de définir pour chaque catégorie de 


\section{TABLEAU I}

Activités considérées dans l'étude du stockage des déchets TFA (Brun-Yaba et al., 1995). Activities looked at in the study of the very low-level waste repository (Brun-Yaba et al., 1995).

\begin{tabular}{cccccc}
\hline Radionucléides & \multicolumn{2}{c}{ Activités } & Radionucléides & \multicolumn{2}{c}{ Activités } \\
& $\left(\mathbf{B q q} \mathbf{g}^{-1}\right)$ & \multicolumn{1}{c}{$\mathbf{T B q}$} & & $\left(\mathbf{B q ~ g}^{-1}\right)$ & $\mathbf{T B q}^{-1}$ \\
\hline${ }^{3} \mathrm{H}$ & 0,1 & $7,8 \times 10^{-2}$ & ${ }^{90} \mathrm{Sr}$ & 0,5 & $5,5 \times 10^{-1}$ \\
${ }^{54} \mathrm{Mn}$ & 0,8 & $4,5 \times 10^{-1}$ & ${ }^{137} \mathrm{Cs}$ & 0,7 & $8,3 \times 10^{-1}$ \\
${ }^{58} \mathrm{Co}$ & 2,2 & 2,1 & ${ }^{239} \mathrm{Pu}$ & 0,1 & $1,2 \times 10^{-1}$ \\
${ }^{60} \mathrm{Co}$ & 2,2 & 2,1 & ${ }^{234} \mathrm{U}$ & 0,5 & $7,3 \times 10^{-1}$ \\
${ }^{63} \mathrm{Ni}$ & 0,5 & $5,0 \times 10^{-1}$ & ${ }^{238} \mathrm{U}$ & 0,5 & $7,3 \times 10^{-1}$ \\
\hline
\end{tabular}

déchets TFA, des filières adaptées, approuvées et contrôlées respectant au mieux les exigences de traçabilité.

\section{Impact potentiel des stockages de déchets TFA}

À l'heure actuelle, le stockage constitue la voie privilégiée d'élimination des déchets radioactifs. Il représente une filière d'élimination à part entière, mais peut aussi constituer l'étape ultime d'une filière de valorisation d'un déchet primaire.

\subsection{Démarche adoptée}

À titre d'illustration et sur la base d'une étude d'impact générique réalisée à l'IPSN, le présent article propose de mettre en lumière la variabilité associée à l'évaluation de l'impact radiologique d'un stockage de déchets TFA de concept simple (Cessac et al., 1997) :

- stockage des déchets en vrac dans des alvéoles en béton ;

- présence d'une couche argileuse de 5 mètres sous le stockage ;

- mise en place d'une couverture simple après exploitation.

La géométrie du stockage retenu est approximativement de $100 \times 35 \times 7 \mathrm{~m}$, pour une capacité de $860000 \mathrm{t}$ de déchets, soit un volume de $250000 \mathrm{~m}^{3}$, correspondant principalement aux déchets engendrés lors des futurs chantiers de démantèlement des installations du cycle du combustible (bétons, gravats, déchets technologiques, ferrailles). Cette capacité a été estimée pour une durée d'exploitation d'environ 50 ans (Brun-Yaba et al., 1995). Le spectre d'activité retenu est représentatif d'une large gamme de déchets TFA ( $c f$. Tab. I).

Seul un scénario d'évolution normale à long terme, après fermeture et oubli du site est traité dans cet article. Les scénarios de types « intrusion humaine » ou «événements naturels exceptionnels » ne sont pas traités ici. 
Le groupe de référence considéré représente un nombre peu important d'individus vivant en forte autarcie à proximité du stockage ou sur le site même. On suppose que les structures de production (agricoles notamment) ainsi que les réserves disponibles en eau (pompage dans un cours d'eau, par exemple) sont juste suffisantes pour alimenter le groupe. Cette hypothèse de dilution minimale augmente le caractère pénalisant du scénario analysé. Les doses calculées doivent être considérées uniquement comme des indicateurs des niveaux de risque potentiels résultant des processus d'évolution du stockage. Elles n'ont aucun caractère prédictif de ce qui pourrait résulter de l'oubli d'un site d'enfouissement. Toute estimation des conditions de vie dans un avenir lointain revêtirait d'ailleurs un caractère largement spéculatif.

Deux types de transfert sont pris en compte : le transport des radioéléments dans la géosphère jusqu'à un exutoire puis leur migration au sein de la biosphère. Les codes de calculs exploités pour évaluer ces transferts ont été respectivement le code GEOS (Ferry, 1996) et le code ABRICOT (Santucci, 1995), développés à I'IPSN. Pour tenter d'illustrer l'amplitude des variations de la dose, deux méthodes ont été successivement appliquées.

Concernant la migration dans la géosphère, des études monoparamètriques préliminaires ont foumi un aperçu de la sensibilité du calcul (la dose efficace individuelle dans le cas présent) au modèle de transport (Cessac et al., 1997). Les résultats présentés dans cet article ont été obtenus pour trois cas de synthèse combinant les valeurs des paramètres prépondérants mis en évidence. Ainsi est appréciée l'amplitude de variation de la dose, tous paramètres géosphère confondus. À chacun de ces cas est associé un degré de réalisme suivant les valeurs retenues ( $c f$. Tabs. II et III).

Concernant les transferts dans la biosphère, les paramètres les plus représentatifs de la dose calculée ont été mis en évidence par une analyse de sensibilité basée sur une analyse de variance. $L$ 'incertitude sur la dose a ensuite été évaluée par analyse statistique de la variabilité de la dose calculée pour différents jeux de valeurs de ces paramètres, générés par hypercube latin. Dans le cas du tritium, on suppose qu'il est en solution dans l'eau et que tous les compartiments de la biosphère sont en équilibre entre eux. En absence d'un modèle plus précis, le tritium n'a pas été pris en compte dans l'évaluation des incertitudes liées au transfert dans la biosphère.

\subsection{Résultats}

L'influence des paramètres caractéristiques du stockage et de la géosphère sur la dose est présentée sur la figure 1, au travers des trois cas décrits plus haut. 


\section{GESTION DES DÉCHETS TFA}

\section{TABLEAU II}

Hypothèses sur les transferts dans la géosphère et caractéristiques des cas de synthèse. Hypothesis on the migration in the geosphere and characteristics of the synthesis cases.

\section{Hypothèses sur le transport dans la géosphère}

- Transport 1D dans la zone insaturée - Prise en compte uniquement de la rétention par l'intermédiaire d'un coefficient de distribution $(\mathrm{Kd})$ aquifere.

- Transport 2D dans l'aquifère par advection et dispersion cinématique (prise en compte uniquement de la rétention par l'intermédiaire $\mathrm{Kd}$ ).

- Pour le tritium, absence de rétention et migration à la même vitesse que l'eau dans la géosphère.

\begin{tabular}{|c|c|c|}
\hline Cas pessimiste & Cas moyen & Cas optimiste \\
\hline $\begin{array}{l}\text { - des valeurs faibles du Kd } \\
\text { aquifere }\end{array}$ & $\begin{array}{l}\text { - des valeurs moyennes de Kd } \\
\text { aquifere }\end{array}$ & $\begin{array}{l}\text { - des valeurs fortes de Kd } \\
\text { aquifere }\end{array}$ \\
\hline $\begin{array}{l}\text { - des valeurs élevées de la Frac- } \\
\text { tion Annuelle Lixiviée (FAL) }\end{array}$ & $\begin{array}{l}\text { - des valeurs faibles de la FAL } \\
\text { (déchets non enrobés) puis fortes à }\end{array}$ & $\begin{array}{l}\text { - des valeurs faibles de la FAL } \\
\text { (déchets enrobés) }\end{array}$ \\
\hline $\begin{array}{l}\text { - une vitesse d'écoulement dans } \\
\text { la nappe de } 400 \mathrm{~m} / \mathrm{an}\end{array}$ & $\begin{array}{l}\text { partir de } 1000 \text { ans (ouvrages } \\
\text { dégradés) }\end{array}$ & $\begin{array}{l}\text { - une vitesse d'écoulement dans } \\
\text { la nappe de } 100 \mathrm{~m} / \mathrm{an}\end{array}$ \\
\hline $\begin{array}{l}\text { - une distance à l'exutoire de } \\
100 \mathrm{~m}\end{array}$ & $\begin{array}{l}\text { - une vitesse d'écoulement dans la } \\
\text { nappe de } 100 \mathrm{~m} / \mathrm{an}\end{array}$ & $\begin{array}{l}\text { - une distance à l'exutoire de } \\
500 \mathrm{~m}\end{array}$ \\
\hline $\begin{array}{l}\text { - prise en compte d'une couche } \\
\text { argileuse de } 5 \text { mètres } \\
\text { d'épaisseur sous le stockage } \\
\text { (zone insaturée) }\end{array}$ & $\begin{array}{l}\text { - une distance à l'exutoire de } 250 \mathrm{~m} \\
\text { - prise en compte d'une couche } \\
\text { argileuse de } 5 \text { mètres d'épaisseur } \\
\text { sous le stockage (zone insaturée) }\end{array}$ & $\begin{array}{l}\text { - prise en compte d'une couche } \\
\text { argileuse de } 5 \text { mètres } \\
\text { d'épaisseur sous le stockage } \\
\text { (zone insaturée) }\end{array}$ \\
\hline $\begin{array}{l}\text { - loi normale de dégradation } \\
\text { des bétons sur } 300 \text { ans } \\
\mathrm{N}(\sigma=45 \text { ans, } \tau=150 \text { ans })\end{array}$ & $\begin{array}{l}\text { - loi normale de dégradation des } \\
\text { bétons sur } 1000 \text { ans } N(\sigma=170 \text { ans, } \\
\tau=500 \text { ans })\end{array}$ & $\begin{array}{l}\text { - loi normale de dégradation des } \\
\text { bétons sur } 1000 \text { ans } \\
N(\sigma=170 \text { ans, } \tau=500 \text { ans })\end{array}$ \\
\hline
\end{tabular}

\section{TABLEAU III}

Valeurs de Kd et de FAL (fraction annuelle lixiviée) considérées (par an). Distribution coefficient and leached activity fraction values (per year).

\begin{tabular}{|c|c|c|c|c|c|c|}
\hline \multirow[t]{2}{*}{ Radionucléides } & \multicolumn{3}{|c|}{$\begin{array}{c}\text { Kd aquifere } \\
\left(\mathrm{m}^{3} \mathrm{~kg}^{-1}\right)(\text { AIEA, 1994) }\end{array}$} & \multirow{2}{*}{ 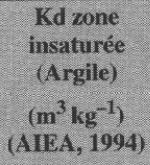 } & \multicolumn{2}{|c|}{$\begin{array}{l}\text { Fraction Annuelle } \\
\text { Lixiviée } \\
\text { FAL }\left(\mathrm{an}^{-1}\right)\end{array}$} \\
\hline & pessimiste & moyen & optimiste & & $\begin{array}{l}\text { colis } \\
\text { intègre }\end{array}$ & $\begin{array}{l}\text { colis } \\
\text { dégradé }\end{array}$ \\
\hline${ }^{3} \mathrm{H}$ & 0 & 0 & $\overline{0}$ & 0 & $2 \times 10^{-3}$ & 0,2 \\
\hline${ }^{54} \mathrm{Mn}$ & $3,0 \times 10^{-3}$ & $4,9 \times 10^{-2}$ & $8,1 \times 10^{-1}$ & $3,3 \times 10^{-3}$ & $4 \times 10^{-4}$ & $4 \times 10^{-2}$ \\
\hline${ }^{58} \mathrm{Co}$ & $2,2 \times 10^{-4}$ & $6,0 \times 10^{-2}$ & 16,0 & 7,3 & $4 \times 10^{-4}$ & $4 \times 10^{-2}$ \\
\hline${ }^{60} \mathrm{Co}$ & $2,2 \times 10^{-4}$ & $6,0 \times 10^{-2}$ & 16,0 & 7,3 & $4 \times 10^{-4}$ & $4 \times 10^{-2}$ \\
\hline${ }^{63} \mathrm{Ni}$ & $2,0 \times 10^{-2}$ & $6,7 \times 10^{-2}$ & 8,1 & $1,6 \times 10^{-1}$ & $4 \times 10^{-4}$ & $4 \times 10^{-2}$ \\
\hline${ }^{90} \mathrm{Sr}$ & $5,5 \times 10^{-4}$ & $1,3 \times 10^{-2}$ & $3,3 \times 10^{-1}$ & $2,0 \times 10^{-3}$ & $10^{-3}$ & 0,1 \\
\hline${ }^{137} \mathrm{Cs}$ & $1,8 \times 10^{-3}$ & $2,7 \times 10^{-1}$ & 40,0 & $7,4 \times 10^{-2}$ & $2 \times 10^{-3}$ & 0,2 \\
\hline${ }^{239} \mathrm{Pu}$ & $1,8 \times 10^{-2}$ & $5,4 \times 10^{-1}$ & 16,0 & $7,4 \times 10^{-2}$ & $10^{-3}$ & 0,1 \\
\hline${ }^{234} \mathrm{U}$ & $5,5 \times 10^{-5}$ & $3,3 \times 10^{-2}$ & 20,0 & $4,0 \times 10^{-3}$ & $6 \times 10^{-5}$ & $6 \times 10^{-3}$ \\
\hline${ }^{238} \mathrm{U}$ & $5,5 \times 10^{-5}$ & $3,3 \times 10^{-2}$ & 20,0 & $4,0 \times 10^{-3}$ & $6 \times 10^{-5}$ & $6 \times 10^{-3}$ \\
\hline
\end{tabular}




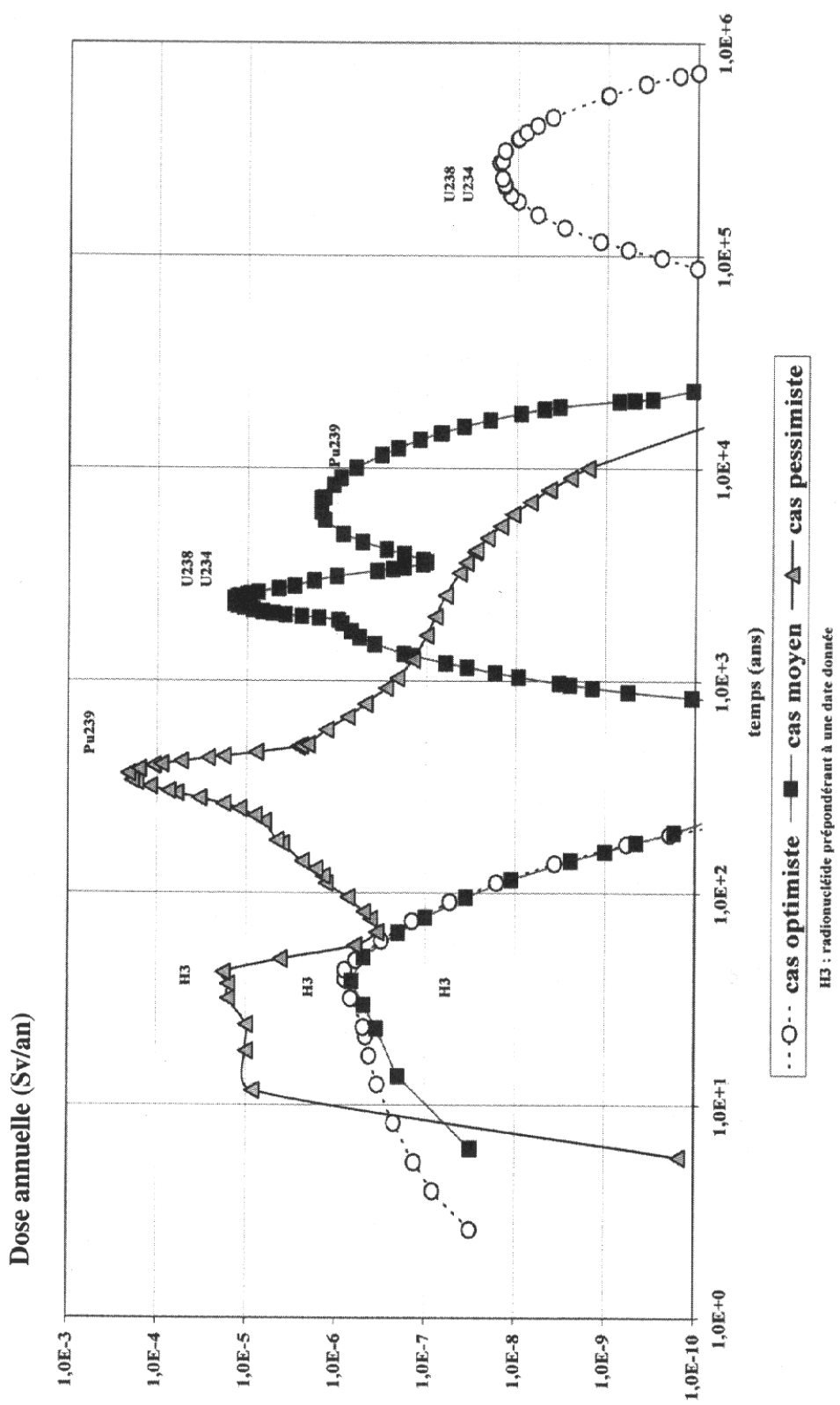




\section{TABLEAU IV}

Incertitudes globales sur la dose individuelle $\left(\mathrm{Sv} \mathrm{an}^{-1}\right)$ associée au scénario d'évolution normale.

Overall uncertainties regarding the individual dose $\left(\mathrm{Sv} \mathrm{year}^{-1}\right)$ associated with the normal change scenario.

\begin{tabular}{|c|c|c|c|c|}
\hline & \multirow{2}{*}{$\begin{array}{l}\text { Incertitudes } \\
\text { sur la dose } \\
\text { individuelle } \\
\text { (Sv an }{ }^{-1} \text { ) }\end{array}$} & \multicolumn{3}{|c|}{ Paramètres géologiques et conceptuels } \\
\hline & & cas pessimiste & cas moyen & cas optimiste \\
\hline \multirow{3}{*}{$\begin{array}{l}\text { Paramètres } \\
\text { biosphère }\end{array}$} & $\begin{array}{l}\text { borne supérieure des } \\
\text { incertitudes }\end{array}$ & $1,0 \times 10^{-3}$ & $5,5 \times 10^{-5}$ & $8,2 \times 10^{-8}$ \\
\hline & calcul déterministe & $2,0 \times 10^{-4}$ & $1,1 \times 10^{-5}$ & $1,6 \times 10^{-8}$ \\
\hline & $\begin{array}{l}\text { borne inférieure des } \\
\text { incertitudes }\end{array}$ & $1,7 \times 10^{-5}$ & $1,0 \times 10^{-6}$ & $1,5 \times 10^{-9}$ \\
\hline
\end{tabular}

Le premier pic intervenant après quelques dizaines d'années correspond à un rejet de tritium, quel que soit le cas considéré. La dose correspondant au cas le plus pessimiste atteint un maximum de $2,0 \times 10^{-4} \mathrm{~Sv} \mathrm{an}^{-1}$, à comparer au $1,6 \times 10^{-8} \mathrm{~Sv} \mathrm{an}^{-1}$, maximum de dose, hors tritium, atteint dans le cas le plus optimiste $\left(7,9 \times 10^{-7} \mathrm{~Sv} a^{-1}\right.$, tritium compris $)$. La gamme de variation maximale de la dose atteint quatre ordres de grandeur.

Il ressort que la variabilité des paramètres a une influence d'une part sur la concentration des rejets à l'exutoire, et donc sur la dose associée, et d'autre part sur la répartition dans le temps de ces rejets. Deux phénomènes interviennent de façon prépondérante :

- la description de la dégradation du terme source, au travers des valeurs de la Fraction Annuelle Lixiviée (FAL),

- la rétention des radionucléides dans l'aquifère, symbolisée par un coefficient de distribution, le $\mathrm{Kd}_{\text {aquifère }}$.

Dans le cas du tritium, les différences observées sont dues aux valeurs de la vitesse d'écoulement dans la nappe et de la distance à l'exutoire considérées.

Concernant les transferts dans la biosphère, l'évaluation des incertitudes s'est appuyée sur les calculs déterministes de dose correspondant aux trois cas de synthèses décrits précédemment. Le tableau IV présente la combinaison des incertitudes ainsi obtenues avec celles liées à la modélisation de la géosphère, permettant ainsi d'estimer l'incertitude globale associée au scénario d'évolution normale.

L'amplitude de variation de la dose atteint deux ordres de grandeur du fait des incertitudes biosphère et quatre ordres de grandeur dans le cas de la géosphère. 
La combinaison des incertitudes globales conduit à une dose individuelle comprise entre $1,0 \times 10^{-3}$ et $1,5 \times 10^{-9} \mathrm{~Sv}^{-1}$, hors tritium.

Pour le cas pessimiste, les valeurs de la FAL correspondent à des propriétés dégradées de la couverture et du conditionnement des déchets. De même, les valeurs de $\mathrm{Kd}$ choisies traduisent une rétention faible dans les couches sousjacentes au stockage. Cette situation semble peu vraisemblable étant donnés les principes envisagés par l'ANDRA en matière de géologie (implantation dans une couche argileuse homogène et à fort pouvoir de rétention), de concept (stockage en alvéole) et de mesures de surveillance pour le futur site de stockage dédié aux déchets TFA. À ce titre, nous retiendrons pour l'incertitude sur la dose individuelle, une plage plus réaliste comprise entre $5,5 \times 10^{-5} \mathrm{~Sv}^{-1}$ (cas moyen) et $1,5 \times 10^{-9} \mathrm{~Sv} \mathrm{an}^{-1}$ (cas optimiste).

\section{Filières d'élimination}

Dans le présent article, seules sont abordées les filières hors domaine nucléaire. Des exemples de filières industrielles potentiellement envisageables pour l'élimination ou la valorisation de déchets TFA issus des installations nucléaires d'Électricité de France, tels que des huiles usagées (Cessac, 1997) ou des déchets amiantifères et de calorifuges (Cessac, 1998), sont présentés dans ce paragraphe. La figure 2 présente les différentes étapes des filières considérées, depuis la sortie du déchet de l'INB jusqu'à la valorisation d'un produit fini recyclé dans le domaine public, ou le stockage d'un déchet ultime. Les processus et les postes de travail décrits par la suite ont été renseignés à partir d'informations recueillies auprès d'exploitants d'installations traitant des déchets conventionnels comparables aux déchets TFA considérés ici.

\subsection{Exemple des huiles usagées TFA}

Le stock d'huiles usagées considéré dans l'étude s'élève à $860 \mathrm{~m}^{3}$, comprenant environ trois quarts d'huiles noires, et un quart d'huiles claires. Ces dernières sont préférentiellement recyclées alors que les huiles noires sont valorisées en cimenterie ou comme combustible pour l'incinération d'autres déchets.

Le tableau $\mathrm{V}$ présente les valeurs extrêmes d'activité massique retenues pour les différents radionucléides considérés dans l'étude d'impact.

\subsection{Exemple des déchets amiantifêres et des calorifuges TFA}

Le stock de déchets amiantifères et de calorifuges à inerter, entreposés sur les INB d'EDF en démantèlement, s'élève à 586 tonnes, réparties sur 5 sites différents, 


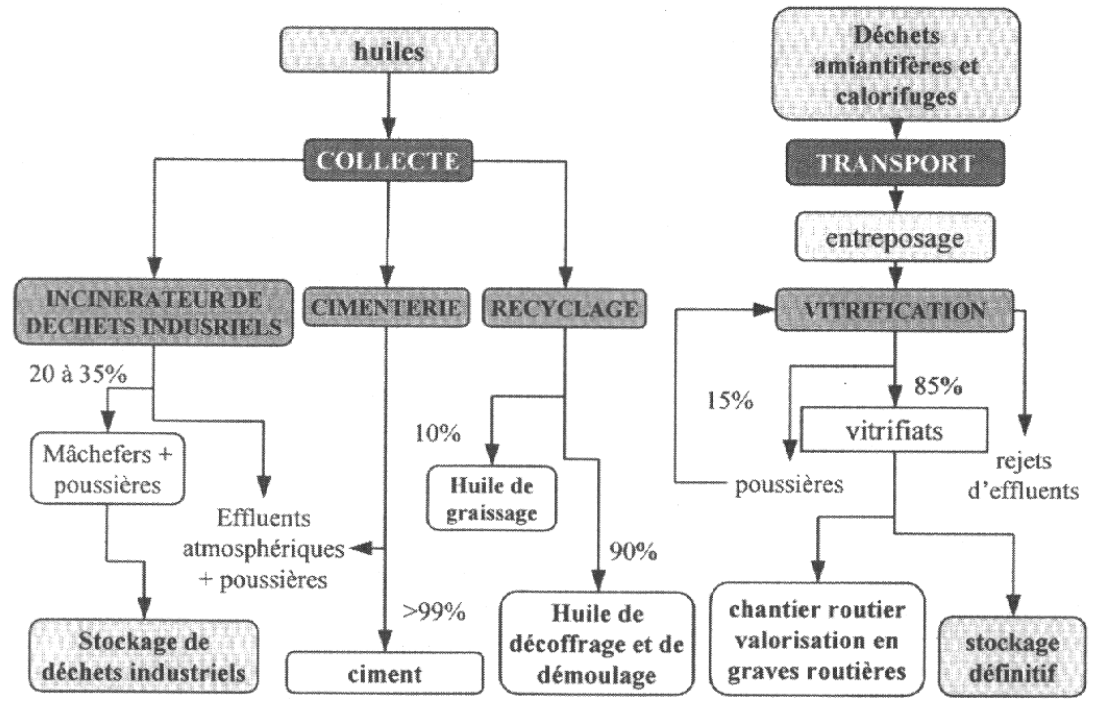

Figure 2 - Filières de traitement des huiles usagées et des déchets amiantifères ou des calorifuges.

Treatment of waste oils and wastes containing asbestos or heat insulating materials.

avec des quantités allant de 3 à 400 tonnes. Quatre radionucléides ont été mis en évidence dans les déchets : le ${ }^{60} \mathrm{Co}$, le ${ }^{137} \mathrm{Cs}$, le ${ }^{134} \mathrm{Cs}$ et le ${ }^{54} \mathrm{Mn}$, à des niveaux d'activité massique totale compris entre $2 \mathrm{~Bq} \mathrm{~g}^{-1}$ et $10 \mathrm{~Bq} \mathrm{~g}^{-1}$. Les différents spectres considérés sont présentés dans le tableau VI.

\subsection{Scénarios d'exposition}

Les scénarios étudiés concernent aussi bien le milieu professionnel ${ }^{1}$ hors nucléaire que le domaine public. Ils prennent en compte les principales voies d'exposition pouvant induire un impact radiologique pour le personnel des installations de traitement ou pour des individus du public, soit :

- exposition externe au contact ou à distance de la source étudiée ;

- exposition interne, par inhalation ou ingestion de substances contaminées.

Concernant les expositions professionnelles, les scénarios ont été établis à partir de la description des postes de travail pour lesquels un contact, direct ou indirect

Dans la suite de l'étude, la dénomination « exposition professionnelle » fait référence au personnel des installations de traitement hors nucléaire, au sens du décret du 2 octobre 1986 (Journal officiel, 1986) relatif à la protection des travailleurs contre les rayonnements ionisants hors INB, en France. 


\section{TABLEAU V}

Activités massiques $\left(\mathrm{Bq}^{-1}\right)$ des radionucléides mis en évidence dans les huiles TFA (Cessac, 1997b).

Specific activities $\left(\mathrm{Bq} \mathrm{g}^{-1}\right)$ of the radionuclides in VLL (very low level) waste oils (Cessac, 1997b).

\begin{tabular}{|cccccc}
\hline Radionucléides & Min. $\left(\mathrm{Bq} \mathrm{g}^{-1}\right)$ & $\begin{array}{c}\text { Max, } \\
\left(\mathbf{B q g} \mathbf{g}^{-1}\right)\end{array}$ & Radionucléides & $\begin{array}{c}\text { Min. } \\
\left(\mathbf{B q ~ g} \mathbf{~ g}^{-1}\right)\end{array}$ & $\begin{array}{c}\text { Max. } \\
\left(\mathbf{B q ~ g ^ { - 1 }}\right)\end{array}$ \\
\hline${ }^{3} \mathrm{H}$ & 0,5 & 0,5 & $110 \mathrm{~m} \mathrm{Ag}$ & 0,1 & 1,2 \\
${ }^{54} \mathrm{Mn}$ & $1,7 \times 10^{-3}$ & $1,9 \times 10^{-2}$ & ${ }^{124} \mathrm{Sb}$ & $8,0 \times 10^{-3}$ & $8,0 \times 10^{-2}$ \\
${ }^{58} \mathrm{Co}$ & $6,6 \times 10^{-2}$ & 0,7 & ${ }^{134} \mathrm{Cs}$ & $1,0 \times 10^{-2}$ & $9,4 \times 10^{-2}$ \\
${ }^{60} \mathrm{Co}$ & 0,3 & 3,6 & ${ }^{137} \mathrm{Cs}$ & $7,2 \times 10^{-2}$ & 0,8 \\
\hline
\end{tabular}

TABLEAU VI

Radionucléides mis en évidence dans les déchets amiantifères TFA (Cessac, 1998). Radionuclides occurring in VLL (very low level) waste containing asbestos (Cessac, 1998).

\begin{tabular}{|c|c|c|c|c|}
\hline Sites & ${ }^{60} \mathrm{Co}$ & ${ }^{137} \mathrm{Cs}$ & ${ }^{134} \mathrm{Cs}$ & ${ }^{54} \mathrm{Mn}$ \\
\hline Brennilis & $100 \%$ & & & \\
\hline Bugey & $98 \%$ & $2 \%$ & - & - \\
\hline Chinon & $95 \%$ & - & - & $5 \%$ \\
\hline Chooz & $14 \%$ & $83 \%$ & $3 \%$ & - \\
\hline Saint-Laurent des Eaux & $90 \%$ & $10 \%$ & - & 4 \\
\hline
\end{tabular}

avec les déchets, ou bien leur proximité, est susceptible de conduire à une exposition radiologique des travailleurs. Ils couvrent les opérations de traitement et de manutention correspondant à une exploitation normale de la filière, ainsi que quelques situations accidentelles concernant les opérations professionnelles.

Le tableau VII présente les scénarios considérés dans l'étude de chacune des filières, en situation normale.

Aucune dilution n'a été considérée par mélange avec d'autres déchets d'origine conventionnelle, avant le traitement. Seuls les processus de concentration ou de dilution liés au procédé sont considérés, comme par exemple le mélange, dans les fours de cimenterie, des résidus de combustion des huiles avec la matière première servant à la fabrication du ciment.

\subsection{Résultats}

Les évaluations réalisées lors des deux études montrent que la voie d'exposition principale est de loin l'exposition externe, due à la présence de ${ }^{60} \mathrm{Co}$ et dans une moindre mesure de ${ }^{137} \mathrm{Cs}$ ou de ${ }^{110 \mathrm{~m}} \mathrm{Ag}$ dans le cas des huiles.

Le tableau VIII présente un comparatif de l'impact radiologique individuel associé aux différents postes de travail des deux filières considérées. Pour chaque 


\section{TABLEAU VII}

Exemples de scénarios considérés dans les évaluations.

Examples of scenarios considered in the assessments.

\begin{tabular}{|c|c|c|c|}
\hline Filière & Type d'exposition & Postes de travail ou situations d'exposition & Voie d'atteinte \\
\hline \multirow{3}{*}{ 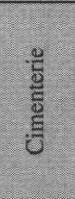 } & \multirow{3}{*}{$\begin{array}{l}\text { exposition } \\
\text { professionnelle } \\
\text { exposition du } \\
\text { public }\end{array}$} & $\begin{array}{l}\text { - exposition à des poussières de ciment } \\
\text { - exposition à un stock de ciment }\end{array}$ & $\begin{array}{l}\text { inhalation, ingestion } \\
\text { exposition externe }\end{array}$ \\
\hline & & $\begin{array}{l}\text { - rejets à la cheminée et captation d'aérosols } \\
\text { par des végétaux ou dépôt sur le sol }\end{array}$ & $\begin{array}{l}\text { inhalation, ingestion } \\
\text { exposition externe }\end{array}$ \\
\hline & & $\begin{array}{l}\text { - séjour dans une maison construite en béton } \\
\text { contaminé }\end{array}$ & \\
\hline \multirow{5}{*}{ 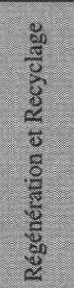 } & \multirow{3}{*}{$\begin{array}{l}\text { exposition } \\
\text { professionnelle }\end{array}$} & - centrifugation & exposition externe \\
\hline & & - manipulation des déchets de procédé & \\
\hline & & - curage des cuves d'entreposage des huiles & \\
\hline & \multirow[t]{2}{*}{$\begin{array}{l}\text { exposition du } \\
\text { public }\end{array}$} & $\begin{array}{l}\text { - utilisation d'huiles recyclées en chantier de } \\
\text { travaux public (décoffrage de structures en } \\
\text { béton) }\end{array}$ & \multirow[t]{2}{*}{$\begin{array}{l}\text { exposition externe } \\
\text { inhalation, ingestion }\end{array}$} \\
\hline & & $\begin{array}{l}\text { - rémanence de l'activité des huiles de } \\
\text { décoffrage dans le béton de construction }\end{array}$ & \\
\hline \multirow{5}{*}{ 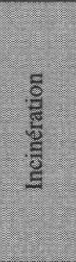 } & \multirow{5}{*}{$\begin{array}{l}\text { exposition } \\
\text { professionnelle } \\
\text { exposition du } \\
\text { public }\end{array}$} & - entretien des installations & \multirow{5}{*}{$\begin{array}{l}\text { inhalation, ingestion } \\
\text { exposition externe } \\
\text { inhalation, ingestion } \\
\text { exposition externe }\end{array}$} \\
\hline & & - transport des mâchefers & \\
\hline & & - stockage des cendres et mâchefers & \\
\hline & & $\begin{array}{l}\text { - rejets à la cheminée et captation d'aérosols } \\
\text { par des végétaux ou dépôt sur le sol }\end{array}$ & \\
\hline & & $\begin{array}{l}\text { - évolution à long terme du stockage: } \\
\text { scénarios d'évolution normale et de type } \\
\text { intrusif }\end{array}$ & \\
\hline \multirow{7}{*}{ 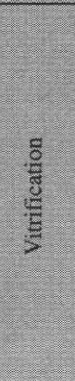 } & \multirow[t]{4}{*}{$\begin{array}{l}\text { exposition } \\
\text { professionnelle }\end{array}$} & $\begin{array}{l}\text { - entreposage (manutention, séjour à } \\
\text { proximité) }\end{array}$ & \multirow[t]{4}{*}{$\begin{array}{l}\text { exposition externe, } \\
\text { inhalation }\end{array}$} \\
\hline & & - contrôle des colis & \\
\hline & & $\begin{array}{l}\text { - exploitation du four et maintenance } \\
\text { (réfractaire, filtres) }\end{array}$ & \\
\hline & & - gestion des vitrifiats & \\
\hline & \multirow[t]{3}{*}{$\begin{array}{l}\text { exposition du } \\
\text { public }\end{array}$} & $\begin{array}{l}\text { - Valorisation des vitrifiats en graves } \\
\text { routières }\end{array}$ & \multirow[t]{3}{*}{$\begin{array}{l}\text { exposition externe, } \\
\text { inhalation, ingestion }\end{array}$} \\
\hline & & $\begin{array}{l}\text { - rejets à la cheminée et captation d'aérosols } \\
\text { par des végétaux ou dépôt sur le sol }\end{array}$ & \\
\hline & & $\begin{array}{l}\text { - cycliste sur une route construite avec des } \\
\text { vitrifiats TFA }\end{array}$ & \\
\hline
\end{tabular}

poste, l'intervalle de dose présenté correspond aux valeurs extrêmes des activités massiques de chacun des spectres retenus. Lorsqu'un tonnage n'est pas spécifié, les valeurs proposées correspondent alors au traitement de la totalité du stock de déchets.

Les résultats obtenus montrent que :

Les opérations de transport et de déchargement entraînent un impact radiologique relativement plus important, que les autres situations d'exposition 


\section{TABLEAU VIII}

Impact $\left(\mu \mathrm{Sv} \mathrm{an}^{-1}\right)$ par poste associé au traitement des huiles usagées et des déchets amiantifères TFA.

Impact $\left(\mu \mathrm{Sv}\right.$ year $\left.^{-1}\right)$ by workstation for treating very low-level waste oils and waste containing asbestos

\begin{tabular}{|c|c|c|c|c|c|}
\hline & \multirow[t]{2}{*}{$\begin{array}{c}\text { Situations } \\
\text { d'exposition }\end{array}$} & \multicolumn{3}{|c|}{ Huiles } & \multirow[t]{2}{*}{$\begin{array}{l}\text { Amiante } \\
\text { Vitrification }\end{array}$} \\
\hline & & Incinération & Cimenterie & Recyclage & \\
\hline \multirow{2}{*}{$\frac{5}{\frac{D}{0}}$} & $\begin{array}{l}\text { Transport routier } \\
\text { (1 seul chauffeur) }\end{array}$ & & 11 a 119 & & $\begin{array}{c}3 \text { à } 13 \text { ( } 3 \text { t traitées) } \\
284 \text { a } 1420 \\
\text { (400 } \text { i traitées) }\end{array}$ \\
\hline & Déchargement & & 7 à 68 & & $\begin{array}{c}20 \text { à } 138 \text { (manuel) } \\
2 \text { a } 7 \text { (grue) }\end{array}$ \\
\hline \multirow{7}{*}{ 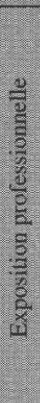 } & $\begin{array}{l}\text { Entreposage avant } \\
\text { traitement }\end{array}$ & - & - & - & $\begin{array}{l}67 \text { à } 333 \text { (intérieur) } \\
9 \text { a } 45 \text { (extérieur) }\end{array}$ \\
\hline & Manutention & - & - & 4 à 44 & 35 à 177 \\
\hline & Exploitation & - & - & 2 à 17 & 1 à 9 \\
\hline & $\begin{array}{l}\text { Entreposage après } \\
\text { traitement }\end{array}$ & $\begin{array}{c}2 \text { à } 21 \\
\text { (mâchefers) }\end{array}$ & $\begin{array}{c}5 \text { à } 55 \\
\text { (ciment) }\end{array}$ & - & 4 à 20 \\
\hline & $\begin{array}{l}\text { Transport des } \\
\text { produits ultimes }\end{array}$ & 4 à 41 & - & - & 2 à 9 \\
\hline & Maintenance & 0,1 à 1 & - & - & 4 à 18 \\
\hline & $\begin{array}{l}\text { Situations } \\
\text { accidentelles }\end{array}$ & $\leq 6$ & dent lors du tra & port) & $\begin{array}{c}\leq 1 \text { (rejets } \\
\text { atmosphériques) }\end{array}$ \\
\hline \multirow{4}{*}{ 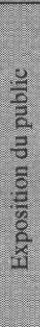 } & Rejets d'effluents & 0,01 à 0.2 & 0,01 à 0,2 & - & 0,03 à 0,2 \\
\hline & $\begin{array}{l}\text { Valorisation d'un } \\
\text { produit ultime }\end{array}$ & - & ciment & $\begin{array}{c}\text { huiles de } \\
\text { décoffrage pour } \\
\text { travaux public }\end{array}$ & $\begin{array}{c}\text { vitrifiats concassés } \\
\text { utilisés comme } \\
\text { graves routières }\end{array}$ \\
\hline & Exposition associée & & $\begin{array}{c}72 \text { à } 760 \\
\text { (construction } \\
\text { en béton } \\
\text { contaminé) }\end{array}$ & $\begin{array}{c}13 \text { à } 130 \\
\text { (rémanence des } \\
\text { huiles de } \\
\text { décoffrage) }\end{array}$ & $\begin{array}{c}1 \text { à } 6 \\
\text { (cycliste sur route) }\end{array}$ \\
\hline & $\begin{array}{l}\text { Stockage d'un } \\
\text { déchet ultime }\end{array}$ & $\begin{array}{c}\leq 0,5 \\
\text { (mâchefers) }\end{array}$ & - & - & $\begin{array}{c}\leq 10^{-3} \\
\text { (vitrifiats) }\end{array}$ \\
\hline
\end{tabular}

professionnelle, avec des doses allant de $10 \mu \mathrm{Sv}^{-1}$ à plus de $1 \mathrm{mSv} \mathrm{an}^{-1}$ (stock de 400 tonnes de déchets amiantifères), quelle que soit la filière considérée. Ceci s'explique par la proximité de la source par rapport au chauffeur et l'absence de protection biologique intercalée, mais aussi par le nombre de rotations important à effectuer étant donnés les forts tonnages de déchets à traiter. Dans le cas du transport de 400 tonnes de déchets amiantifères, environ 30 chauffeurs ou une protection biologique de $6 \mathrm{~cm}$ de plomb seraient nécessaires pour que l'impact individuel soit de l'ordre de $10 \mu \mathrm{Sv} \mathrm{an}^{-1}$ pour une activité massique totale de $2 \mathrm{~Bq} \mathrm{~g}^{-1}$ dans les déchets (impact global de l'opération : $284 \mu \mathrm{Sv} \mathrm{an}^{-1}$ ). Pour une activité massique initiale de $10 \mathrm{~Bq} \mathrm{~g}^{-1}$ (impact global de $1420 \mu \mathrm{Sv} \mathrm{an}^{-1}$ ), 
le nombre de chauffeurs serait porté à plus de 100 et l'épaisseur de la protection a environ $10 \mathrm{~cm}$.

- L'exploitation et la maintenance des installations de traitement entraînent un impact radiologique individuel inférieur ou égal à $10 \mu \mathrm{Sv} \mathrm{an}^{-1}$, pour des activités massiques faibles dans les déchets.

Dans le cas du traitement des déchets amiantifères et des calorifuges, deux opérations se distinguent avec un impact de quelques dizaines à quelques centaines de $\mu$ Sv $a^{-1}$ :

- l'entreposage des déchets, avant traitement ;

- la manutention.

Ces situations entraînent en effet à la fois une proximité à de grandes quantités de déchets et des temps d'exposition cumulés importants. S'agissant de la manutention, c'est surtout la proximité immédiate, voire le contact, sans réelle protection biologique, qui sont la cause des doses les plus élevées.

- L'aval des filières induit des expositions qui découlent de l'utilisation de produits valorisés ou de la gestion des déchets de procédé. C'est à ce niveau qu'apparaît la spécificité de chacune des filières.

Dans le cas des déchets amiantifères et des calorifuges TFA, l'impact sur le public resterait faible, voisin de $10 \mu \mathrm{Sv} \mathrm{an}^{-1}$, que les vitrifiats TFA soient stockés ou valorisés en graves routières.

Concernant les huiles usagées TFA, il apparaît que pour les filières cimenterie et recyclage, l'impact calculé pour ces scénarios est toujours supérieur à $10 \mu \mathrm{Sv} \mathrm{an}^{-1}$, voire à $100 \mu \mathrm{Sv} \mathrm{an}^{-1}$. Les situations prises en compte correspondent à une résidence quasi quotidienne dans une habitation construite entièrement avec des matériaux contaminés.

Le produit sortant de la filière incinération est un déchet ultime qui est expédié en centre de stockage. L'impact associé à ce mode de gestion serait au maximum de $1 \mu \mathrm{Svan}{ }^{-1}$, et interviendrait après une période d'au moins 30 ans, durée minimale de surveillance d'un centre de stockage de déchets industriels.

\section{Conclusion}

Les évaluations d'impact radiologique réalisées sur des stockages génériques de déchets TFA montrent que les incertitudes sont importantes si l'on combine la variabilité des paramètres descriptifs du concept, de la géosphère et de la 
biosphère. Leur réduction est largement dépendante de l'amélioration des connaissances actuelles. En particulier, la définition des futurs sites de stockage devrait permettre de fixer différents points :

- le concept de stockage à prendre en compte : ouvrages, barrières ouvragées... ;

- le conditionnement des déchets ;

- les principes de gestion, à savoir la durée de la période de surveillance et les dispositions administratives mises en æuvre pour le contrôle du site ;

- le site retenu, pour une prise en compte plus réaliste des phénoménologies intervenant dans la migration des radionucléides.

Concernant les filières conventionnelles, les études réalisées ont montré la nécessité de considérer la filière dans sa globalité et de faire des évaluations poste par poste afin de mettre en lumière les situations les plus pénalisantes telles que le transport.

S'agissant d'expositions en milieu professionnel, différentes mesures peuvent être adoptées pour réduire l'exposition radiologique des travailleurs, dans une perspective d'optimisation.

- Dans le cas du transport routier, une augmentation du nombre de chauffeurs ou la mise en place d'une protection biologique permet de réduire l'impact individuel. La pratique d'un transport ferroviaire est une alternative permettant également une nette réduction des doses reçues.

- Les doses reçues lors de la gestion des entreposages de déchets primaires ou ultimes peuvent être évitées ou tout du moins réduites par la mise en place d'un système d'élimination ou d'évacuation des déchets en flux tendus.

- Enfin, la manutention peut être réduite par automatisation de certaines tâches manuelles.

S'agissant d'individus du public exposés par utilisation directe d'un produit valorisé (huiles de décoffrage ou de ciment TFA en chantier de travaux public, par exemple) ou ultérieure (habitation dont les murs sont contaminés), l'application de contre-mesures visant à réduire les doses reçues n'est pas envisageable. Elle serait d'une part contradictoire avec la nécessité d'une absence de risques significatifs (par exemple au-dessus d'une exposition de $10 \mu \mathrm{Sv}_{\mathrm{an}}{ }^{-1}$ pour une pratique donnée) pour ces individus et d'autre part, elle serait extrêmement complexe à mettre en œuvre du fait de la difficulté à maintenir la traçabilité des matériaux potentiellement contaminés en aval de la filière. Dans le cas des huiles usagées TFA, cette constatation conduit certainement à écarter les filières d'incinération en cimenteries et celles de recyclage. Seule la filière d'élimination en incinérateur de déchets industriels semble envisageable. 


\section{RÉFÉRENCES}

Brun-Yaba C., Pérès J.M. (1995) Impact radiologique d'une décharge spécifique de déchets de très faible activité. Rapport interne IPSN.

Cessac B., Badie M., Pérès J.M., Santucci P. (1997) Revue des incertitudes associées aux études d'impact d'un stockage de déchets de très faible activité. Rapport interne IPSN.

Cessac B. (1997) Étude de l'impact radiologique de quelques filières industrielles envisagées pour la gestion des huiles usagées TFA entreposées sur les sites INB d'EDF. Rapport interne IPSN.

Cessac B. (1998) Étude de l'impact radiologique de la filière d'inertage par vitrification des déchets amiantifères de très faible activité. Rapport interne IPSN.

DSIN (1997) La gestion des déchets très faiblement radioactifs - "Contrôle " - revue de l'autorité de sûreté nucléaire. Direction de la sûreté des installations nucléaires $n^{\circ} 118$, Paris.

Ferry C. (1996) Le code GEOS, Version 2.0 : Calcul des transferts dans la géosphère - Théorie et utilisation. Rapport interne IPSN.

Hilmoine R. (1997) Les filières de déchets de très faible activité à EDF. In : Actes de La gestion des déchets de très faible activité : enjeux et impacts, 13-14 novembre 1997, Société française de radioprotection.

IAEA (1994) Handbook of parameter values for the prediction of radionuclide transfer in temperate environments. Technical reports series $\mathrm{n}^{\circ} 364$. IAEA, Vienna.

Journal officiel (1986) Décret $n^{\circ} 86-1103$ du 2 octobre 1986, relatif à la protection des travailleurs contre les dangers des rayonnements ionisants. Journal Officiel du 10 octobre 1986, Paris.

Santucci P. (1995) ABRICOT : conceptual and mathematical modelling of Biosphere - version 2.0. Rapport interne IPSN. 